\title{
Interpretation of the features of a linear regression model for predicting the survival time of the amyotrophic lateral sclerosis patients with mutated SOD1
}

\author{
Nikolay Alemasov \\ The Federal Research Center \\ Institute of Cytology and Genetics \\ The Siberian Branch of the Russian \\ Academy of Sciences \\ Novosibirsk, Russia \\ ORCID iD: 0000-0002-7511-5385
}

\author{
Alexandr Shcherbakov \\ Novosibirsk State Technical University \\ Novosibirsk, Russia \\ aleksandr.serbakov@yandex.ru \\ Vladimir Timofeev \\ Novosibirsk State Technical University \\ Novosibirsk, Russia \\ v.timofeev@corp.nstu.ru
}

\author{
Vladimir Ivanisenko \\ The Federal Research Center \\ Institute of Cytology and Genetics \\ The Siberian Branch of the Russian \\ Academy of Sciences \\ Novosibirsk, Russia \\ salix@bionet.nsc.ru
}

\begin{abstract}
Amyotrophic lateral sclerosis (ALS) is an incurable neurodegenerative disease characterised by the inevitable degeneration of central and peripheral motor neurons. Aggregation of mutant SOD1 is one of the molecular mechanisms underlying the onset of the disease. Previously, we proposed regression models linking the change in the stability of hydrogen bonds in mutant SOD1 calculated using molecular dynamics with patients' survival time. In this work, we developed an approach to the interpretation of features of linear regression models with the aim of a deeper understanding of the structural effects of mutations in the SOD1 protein. The approach suggested was based on the principal component analysis and over-representation of features including the sequence position of amino acid residues forming the hydrogen bonds detected within a range of the structural elements of the SOD1. This study can help one to deduce important structural regions in the protein which could be further targeted by small chemical compounds.
\end{abstract}

Index Terms - ALS, SOD1, hydrogen bonds, regression models, PCA, over-representation

\section{Introduction}

Amyotrophic lateral sclerosis (ALS) is an incurable neurodegenerative disease [1]. It is known that the second most common and widely studied cause of the familial form of ALS are mutations in the SOD1 gene, which encodes superoxide dismutase-1 [2]. One of the hypotheses regarding the mechanism at the molecular level of the disease is an aggregation of the SOD1 protein induced by its incorrect folding as a result of mutations [2].

Previously, we proposed a regression models for predicting the survival time of patients with ALS on the basis of the analysis of the change in the stability of hydrogen bonds in SOD1 mutants compared to the wildtype protein [3]. The stability of hydrogen bonds was estimated using the analysis of molecular dynamics (MD) trajectories.

In this work, we aimed at interpretation of features included into linear regression models to deeper understand the structural effects of mutations in the SOD1 protein. For this to achieve an approach was suggested based on the principal component analysis and over-representation of positions of multiple features such as the hydrogen bonds detected previously.

\section{Methods}

The stability of the hydrogen bonds between protein atoms and atoms of water molecules and the stability of water bridges from MD trajectories were taken from our previous paper [3]. For the MD simulation AMBER 12 software suite was applied there [4] and hydrogen bonds were detected with the cpptraj utility for each conformation via AmberTools 13 [5].

The observed survival time of patients with different mutations in the SOD1 protein was obtained from the literature [6]. These data was used to construct multiple regression model. To isolate latent factors, the principal component analysis was used, followed by varimax rotation. Later, latent factors were used as independent variables in constructing regression models, the response of which was the survival time of ALS patients. In addition to the classical least-squares method, robust M-estimates were used based on the Huber loss function to construct regression models. There were 100 bootstrap steps with each estimating the standard error (RMSE) as the mean square difference between the predicted and published survival times.

An analysis of principal components was also applied to unravel the internal structure of hydrogen bonds stability formed in the different SOD1 mutants. Over-representation of amino acid residues within different structural regions was calculated with scipy [7].

The several structural regions were considered in the overrepresentation analysis (see e.g. reference [8]).

\section{Results}

As a result of the simulation of the wild-type SOD1 and its mutants 6381 hydrogen bonds were detected in the MD trajectories. However the best RMSE (5.44 years) was for the regression model with only 1545 hydrogen bonds left after filtering those $\mathrm{H}$-bonds with stability less than 0.1 . 


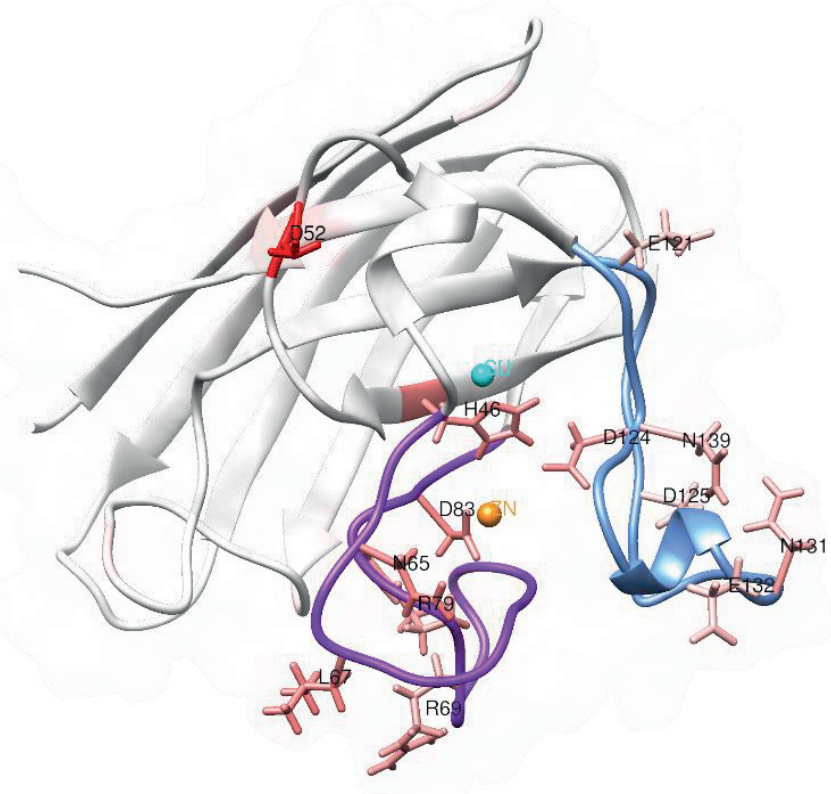

Fig. 1. Spatial structure of the SOD1 monomer with over-represented aminoacid residues shown

The results of evaluating the over-representation in figure 1 . The best regression model using the robust Mestimates was logistic one resulting in the RMSE of 3.59 years (see table I).

TABLE I - RMSE OF THE REGRESSION MODELS BASED ON MESTIMATOR AND LEAST-SQUARE APPROACHES

\begin{tabular}{|c|c|}
\hline Regression model & RMSE, years \\
\hline Logistic based on M-estimator & 3.588 \\
Linear based on M-estimator & 4.19 \\
Cubic based on M-estimator & 5.6 \\
Cubic least-square & 5.75 \\
\hline
\end{tabular}

\section{Discussion}

The literature describes amino acid residues that can participate in the formation of aggregates of SOD1 protein mutants, which are one of the potential factors in the development of the ALS disease [8]. The authors identified regions which involved in the formation of aggregates: the electrostatic loop (residues 121 to 144), the disulfide loop (residues 49-62) and the loop containing the zinc ionbinding site (residues 63-84). According to our results, it was seen that positions $65,67,69,79,83$ from the zincbinding loop and 121, 124-125, 131- 132, 139 from the electrostatic loop known to be involved in aggregation were also involved in the formation of hydrogen bonds from the $\mathrm{PC} 1$. Thus, a change in hydrogen bond stability in these important positions can simultaneously be associated with survival time and affect the aggregation of mutant SOD1.

\section{ACKNOWLEDGMENT}

The authors thank the Siberian Branch of the Russian

Academy of Sciences for the budget project No. 032420190040-C-01 and the Indian Council of Medical Research and Russian Foundation for Basic Research for the joint Project No. 17-54-49004.

\section{REFERENCES}

[1] M. C. Kiernan et al., "Amyotrophic lateral sclerosis", The Lancet, vol. 377 (9769), pp. 942-955, 2011.

[2] E. Tokuda and Y. Furukawa, "Copper Homeostasis as a Therapeutic Target in Amyotrophic Lateral Sclerosis with SOD1 Mutations", International Journal of Molecular Sciences, vol. 17 (5), P. 636, 2016.

[3] N. A. Alemasov et al., "Dynamic properties of SOD1 mutants can predict survival time of patients carrying familial amyotrophic lateral sclerosis", Journal of Biomolecular Structure and Dynamics, vol. 35 (3), pp. 645-656, 2017.

[4] R. Salomon-Ferrer, D. A. Case, and R. C. Walker, "An overview of the Amber biomolecular simulation package", Wiley Interdisciplinary Reviews: Computational Molecular Science, vol. 3 (2), pp. 198-210, 2013.

[5] D. R. Roe, T. E. Cheatham, and T. E. Cheatham III, "PTRAJ and CPPTRAJ: Software for processing and analysis of molecular dynamics trajectory data", Journal of Chemical Theory and Computation, vol. 9 (7), pp. 3084-3095, 2013.

[6] Q. Wang et al., "Protein aggregation and protein instability govern familial amyotrophic lateral sclerosis patient survival", PLoS biology, vol. 6 (7), P. e170, 2008.

[7] P. Virtanen, et al. "SciPy 1.0: fundamental algorithms for scientific computing in Python", Nature Methods, vol. 17, pp. 261-272, 2020

[8] S. Antonyuk et al., "Structural consequences of the familial amyotrophic lateral sclerosis SOD1 mutant His46Arg", in Protein science, vol. 14 (5), pp. 1201-1213, 2005. 